\title{
A NUMERICAL MODEL FOR THE EVALUATION OF GEROTOR TORQUE CONSIDERING MULTIPLE CONTACT POINTS AND FLUID-STRUCTURE INTERACTIONS
}

\author{
Zubin Mistry ${ }^{*}$, Venkata Harish Babu Manne ${ }^{1}$, Andrea Vacca $^{1}$, Etienne Dautry ${ }^{2}$, Martin \\ Petzold $^{2}$ \\ ${ }^{1}$ Maha Fluid Power Research Center, Purdue University, 1500 Kepner Drive, 47905 Lafayette, USA \\ ${ }^{2}$ Thomas Magnete GmbH, Innomotion Park 3, 57562 Herdorf, Germany \\ *Corresponding author: Tel.: +1 7656053090, email address: mistry7@purdue.edu
}

\section{ABSTRACT}

This paper presents a numerical model for the evaluation of the actual torque in Gerotor units. The model consists of two major modules: the pre-processor module and the HYGESim module. The preprocessor module consists of the geometric and the mechanical module. The geometric pre-processor module considers the CAD geometry of Gerotor with tolerances as input and it provides as output the geometric features needed to evaluate the rotor loading and the flow features. The mechanical preprocessor module evaluates the forces of interaction at the contact points between the rotors. The flow displaced by the unit is evaluated using a lumped parameter model whereas the lubricating gaps are evaluated by solving the Reynolds Equation. The main novel aspects consist of the evaluation of the frictional losses at various interfaces. An Elasto-Hydrodynamic Lubrication (EHL) approach is used to evaluate the frictional losses at the contact points between the rotors.

Tests on a prototype Gerotor unit are performed for the model validation, particularly as pertains to the features of the shaft torque. Additionally, the paper comments on the distribution of the different torque loss contributions associated with the operation of the unit taken as reference.

Keywords: Gerotor, Lumped Parameter Model, Multiple Contact Points

\section{INTRODUCTION}

Gerotor pumps are one of the popular types of hydrostatic units in low-pressure fluid-power and automotive applications. Low cost, compactness, robustness, and low noise are some of the factors behind the success of these units. Fig. 1 illustrates a typical assembly of a Gerotor unit which consists of an inner rotor, outer rotor, body, suction, and delivery port.

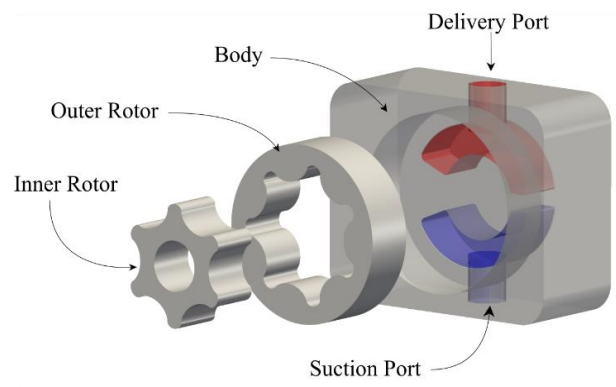

Figure 1: A typical Gerotor assembly
Fig. 2 illustrates the working of a Gerotor: the inner rotor and the outer rotor rotates about a fixed axis separated by a certain eccentricity. The figure shows the changes in pressures in different Tooth Space Volumes (TSVs) with time (where one of the TSV is labelled as $T S V_{i}$ ).
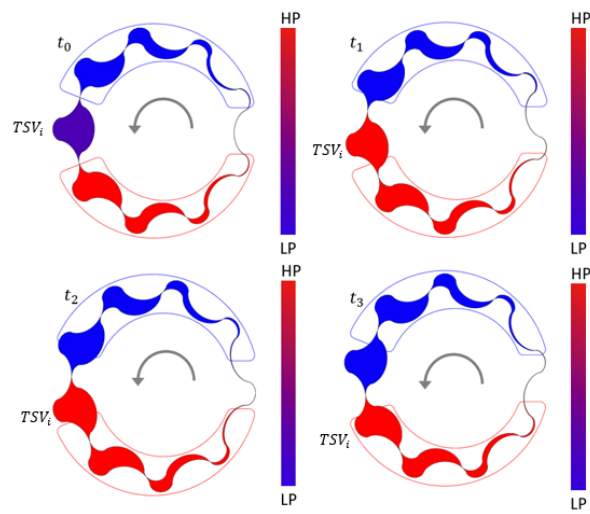

Figure 2: Principle of operation of a Gerotor 
The unit has two primary lubricated interfaces which are shown in Fig. 3. One lubricated region is the radial gap which is a thin fluid film present between the outer rotor and the body. This lubricated interface also acts as a journal bearing. The second lubricated region is the lateral gap which is a thin fluid film present between the lateral surfaces of the gears and the body. This lateral gap is present on both the lateral surfaces of the gears. Fig. 3 shows one of the two lateral gaps.

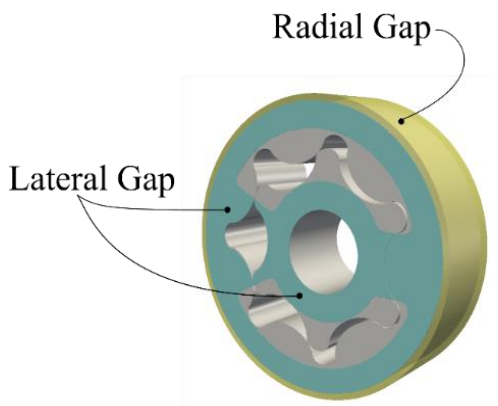

Figure 3: Lubricated interfaces in a Gerotor

Although a Gerotor was first conceived in the 1800 s, recent advances in fluid power and automotive field pushes the limits of the working conditions of the Gerotor by demanding higher operating pressures and higher efficiency. This calls for accurate models that can aid the designers in predicting the behavior of different aspects of Gerotor under different operating conditions.

Various past efforts have taken up this challenging task of modeling the fluid-dynamic behavior of Gerotor units. The first analytical models were proposed by Bonandrini et al. [1,2] where they presented analytical approaches to perform comparative evaluations between the flow parameters given by gears based on hypo trochoidal and epitrochoidal envelopes with different design parameters. Mentioned analytical approaches of study significantly contributed in advancing both the understanding and the modeling of Gerotors; however, they have limitations given by some simplistic assumptions, such as the absence of the clearance between the rotors, of fluid leakages or effects due to fluid compressibility.

Various numerical models have been introduced during the past few decades to describe these effects. Nervegna et al. [3, 4] formulated a control volume lumped parameter model, based on continuity and orifice equations, that can be used for basic studies on the flow displacing of the unit. A similar formulation was extended for a more generic case by Schweiger et al. [5], who included also a more detailed calculation for the internal leakages.

The rise in computational power saw a change in the modeling of a Gerotor unit. Studies of Gerotor units based on commercial CFD tools started to appear highlighting the possibility of showing more detailed flow features such as the work of Gamez-Montero et al. [6] and Altare and Rundo [7]. Simulation time can represent a limit for CFD simulation approaches for optimization studies involving numerous design parameters. Moreover, the need for a continuous fluid domain implies the impossibility of reproducing actual contacts between the rotors.

The recent study by Pellegri et al. [8] has shown a comparison between CFD, lumped parameter model and experimental results showing a close match between experimental, CFD and lumped parameter based model results for different operating conditions. In another research study, Pellegri et al. [9] illustrated the effect of rotor micro-motion on the prediction and experimental validation of flow pulsation and pressure ripple results with an advantage of having lower computational time when compared with traditional CFD based approaches. This study sets a very important base for the current research work as it shows that lumped parameter models are computationally inexpensive when compared to CFD based approaches and can be easily coupled with dynamic models to permit the study of micro-motions of the internal parts.

Much effort was also put by researchers into modeling the fluid dynamics of the Gerotor but less into the contact mechanics of the unit. In this regard, a significant work is the one published by Gamez-Montero et al. [10] where the authors studied the force transmission between the rotors assuming contact at multiple points, comparing an analytical approach for the evaluation of the contact stress between the teeth with a finite element approach under a quasi-static condition. This approach has its limitation that FEM method requires very high computational time and therefore limits its applicability to dynamics models.

However, there is still a gap in the numerical prediction of the torque loss that affects the hydromechanical efficiency of a Gerotor. 
Moreover, the pulsation of the shaft torque, which can affect the sizing and functioning of the prime mover, is a parameter still challenging to determine. This paper tackles these challenges, building upon the model first presented by Pellegri et al. [9], by modeling the possibility of multiple contact points in a Gerotor unit and modeling frictional losses at those contact points using EHL formulation [11]. The model described in the present work includes online coupling of the lumped parameter model with lubricating interfaces with the capability of exchanging flows and imparting forces on the gears including the two-dimensional motion of the gears. Experimental results were also used to permit a fair validation of the simulation results and prove the potentials of the model for accurate studies of Gerotor units.

The following sections of the paper describe the different modules of the proposed model. Section 2 describes the simulation model developed by the authors elaborating on different sub-modules. Section 3 shows a comparison of the current model with experimental results for the torque ripple and overall torque for a simulated Gerotor unit with varying operating conditions.

\section{GEROTOR SIMULATION MODEL}

The model is primarily divided into two major groups i.e. Preprocessor Module and HYGESim: Hydraulic Gear Simulation Tool as shown in Fig. 4. The HYGESim tool will be discussed prior to the preprocessor modules as it would provide the necessary insight for understanding the preprocessor modules. The fluid-dynamic module and a portion of the force module were already published in the references $[8,9]$ but a brief explanation is provided here for completeness. Instead, the other modules represent the novel content of this paper.

The HYGESim tool consists of four major sub-modules. First, a fluid dynamics module is based on a lumped parameter approach that is used to evaluate the displacing action of the tooth space chamber and the main flow through the unit. The information of pressure evaluated by the fluid-dynamic module is passed to a forces module which evaluates the instantaneous forces acting on both rotors. The novel aspect of this model with respect to a first formulation published by the authors in [9] is that the module now relaxes the assumption of a single contact point that was previously made. Another novel aspect is the inclusion of Elasto-Hydrodynamic equations at the contact location. Accurate modeling of the radial and lateral surfaces is also important as they have an impact on torque losses

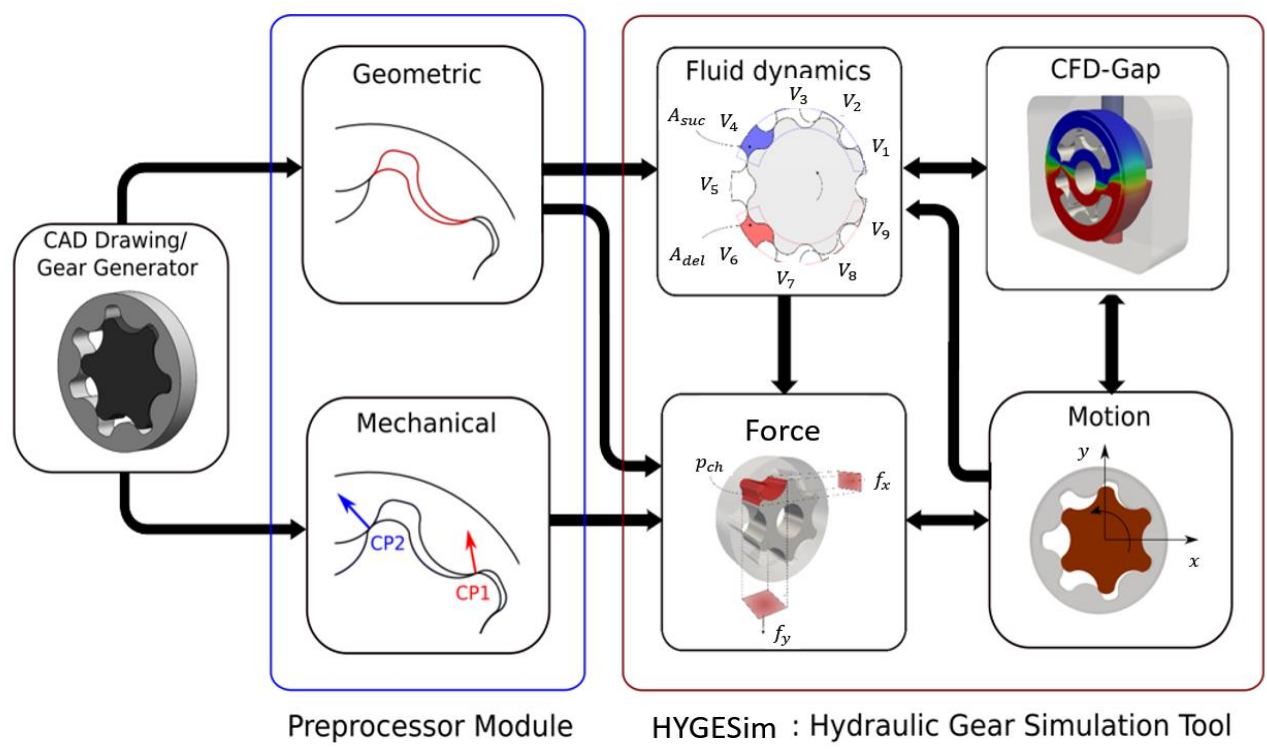

Figure 4: Structure of Gerotor simulation tool 
and fluid leakages. The radial gap and lateral gaps are modeled using CFD-Gap module which solves the Reynolds equation in the film to apply forces to the gears and apply leakages to the connected chambers. All the forces and moments are applied to the gears and are integrated using Newton's laws of motion for a rigid body to generate the position and velocity for the next time step. The HYGESim tool requires a detailed calculation of several geometrical features based on both the rotors' position, a task performed by the geometric module and the mechanical preprocessor module. These modules can read the CAD file details of the unit and pass the information to the HYGESim tool.

\subsection{Fluid Dynamics Module}

The fluid dynamics module represents the core of the proposed simulation tool since all other modules rely on the correct prediction of the pressure distribution in the TSVs. The TSVs have the same properties such as density, pressure, etc. This is the essence of a Lumped Parameter Model. The choice of a lumped parameter approach for this module allows for fast computations and permits easy interfacing with the other sub-modules. The layout of the connections can also be represented with the schematic of Fig 5. The connections between the different Control Volumes (CVs) as well as between CVs and inlet/outlet ports are treated as variable orifices. The instantaneous values for the $\mathrm{CV}$ volumes, the flow area and the equivalent diameter for all the connections are evaluated as a function of the instantaneous shaft angle and the positions of the rotor, by the geometric preprocessor module as it will be clarified in Section 2.5.

Based on the flow between adjacent CVs, mass conservation and fluid state equations, the variation of the pressure $p_{i}$ inside each $\mathrm{CV}$ with respect to time is determined through the pressure build-up equation:

$$
\begin{aligned}
& \frac{d p_{i}}{d t}=\left.\frac{1}{V_{i}} \frac{d p}{d \rho}\right|_{p=p_{i}}\left[\sum \dot{m}_{i n, i}-\sum \dot{m}_{\text {out }, i}-\right. \\
& \left.\left.\rho\right|_{p=p_{i}} \frac{d V_{i}}{d t}\right](\text { with } i=1,2 \ldots . n)
\end{aligned}
$$

In Eq. (1) $V_{i}$ represents the instantaneous volume of the $i^{\text {th }} \mathrm{CV}$. It can also be noted that the bulk modulus $\left.\frac{d p}{d \rho}\right|_{p=p_{i}}$ depends on the fluid properties, particularly on the $p_{i}$ the pressure of the $i^{\text {th }}$ chamber.

The mass flow entering and leaving each $\mathrm{CV}$ ( $\dot{m}_{\text {in }}$ and $\dot{m}_{\text {out }}$ terms in Eq. 1), is evaluated using

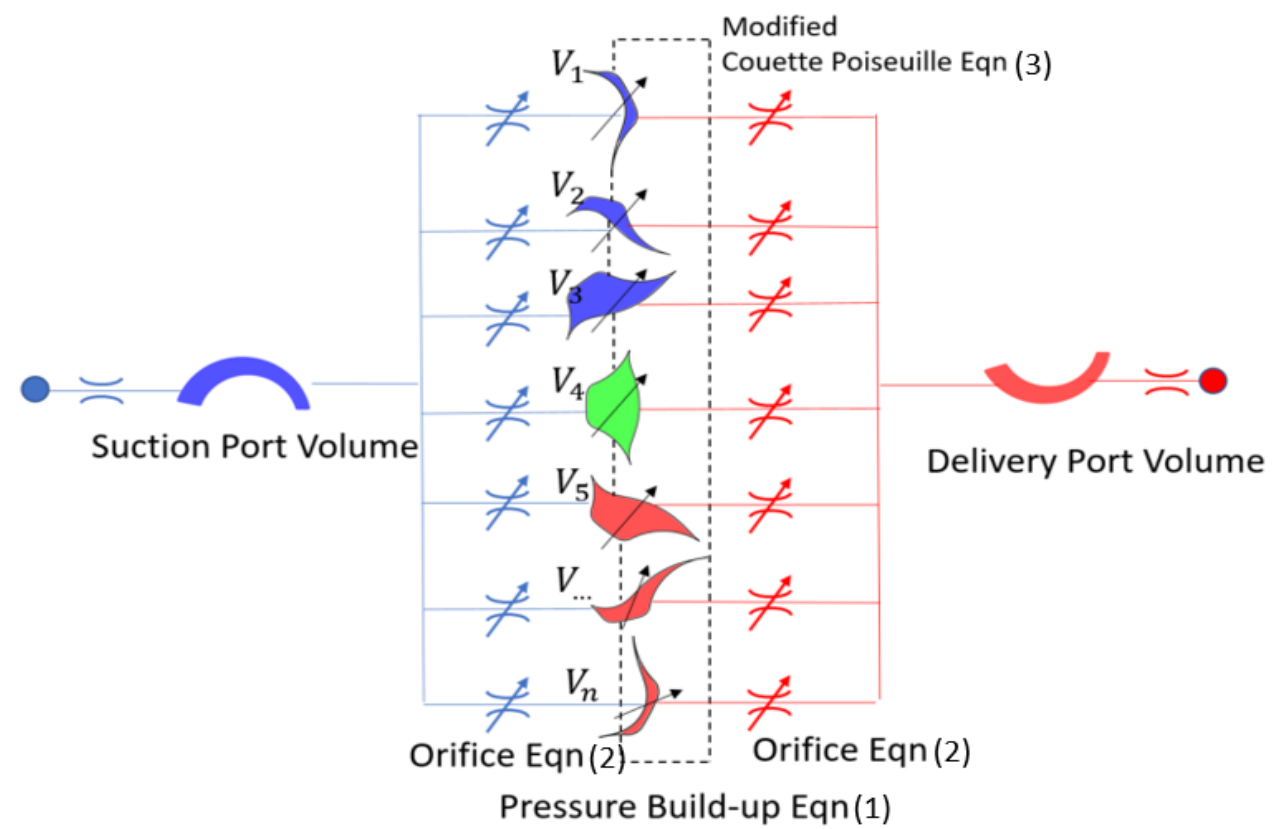

Figure 5: Representation of the control volumes and of the internal connections 
two different approaches: for the connection between each CV and the inlet/outlet ports the orifice equation with a variable flow coefficient $\alpha$ [13] has been used, to account for both the laminar and turbulent regimes. The mass flow rate equation is as follows:

$\dot{m}=\left.\frac{p_{i}-p_{P}}{\left|p_{i}-p_{P}\right|} \cdot \rho\right|_{p=\overline{p_{l, P}}} \cdot \alpha \cdot A_{i, P} \cdot \sqrt{\frac{2 \cdot\left(p_{i}-p_{P}\right)}{\left.\rho\right|_{p=\overline{p_{l, P}}}}}$

Here, the orifice flow coefficient $\alpha$ is a function of Reynolds Number (Re), which is described in [13].

The inter-teeth connection between the different CVs, given by the radial clearance between the rotors (Fig 4.), is treated assuming laminar flow conditions. In the model, the modified Poiseuille equation derived by [14] based on the radius of curvature and gap height (evaluated in Section 2.6) that includes the Couette flow term is used,

$\dot{m}=\rho\left[\frac{0.0456 b h_{t}^{2.4836} \Delta p}{\mu R_{e q}^{0.4836}}+\omega_{\text {outer }} \cdot 2 \pi \cdot r_{\text {outer }}\right.$.

$\left.\frac{h_{t}}{2}-\omega_{\text {inner }} \cdot 2 \pi \cdot r_{\text {inner }} \cdot \frac{h_{t}}{2}\right] \cdot b$

\subsection{Force Module}

Force module evaluates all the forces and moments each gear is subjected to. This module is sub-divided into two sections: the forces due to fluid pressure (already described in the previous work [9]) and the forces due to contact, evaluated using contact mechanics relations including the effects of Elasto-Hydrodynamic Lubrication.

\section{Fluid Pressure Force}

The evaluation of the forces and moments acting on the rotors is based on the instantaneous pressure distribution, given by the pressures in the TSVs, calculated by the fluid dynamic module described in the previous section. The details of this sub-section have been published by Pellegri et. al [9] where for every displacement chamber, the areas of influence of the pressure with respect to the Cartesian coordinates are considered (Fig. 6). Figure 6 illustrates that the forces $F_{x}$ and $F_{y}$, can be evaluated using areas $\Omega_{z y}$ and $\Omega_{z x}$, which assumes a constant pressure, $p_{i}(\theta)$ inside a TSV. The formulation with $X_{i}$ and $Y_{i}$ can be used to evaluate the moment contribution on each rotor. Based on this, the necessary forces and moments are calculated on each gear.

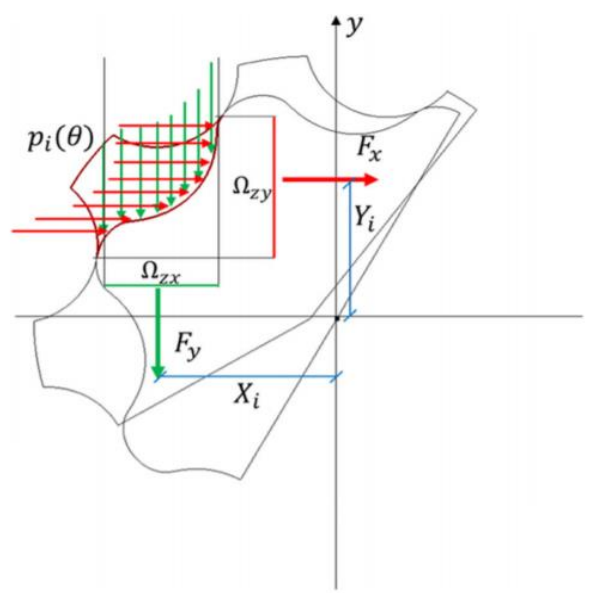

Figure 6: Evaluation of forces and torques [9]

\section{Contact Force}

The contact forces $F_{c}$, as well as the angle of action $\theta$ for each tooth is evaluated based on the look-up table supplied by the mechanical module which will be explained in detail in section 2.6. The contact forces and moment are added to both the gears as shown in Fig. 7. Therefore, the net force and moments on the inner gear can be evaluated as follows:

Net $F_{x, C}=\sum_{i=1}^{n} \mathrm{~F}_{\mathrm{C}_{\mathrm{i}}} \cos \left(\theta_{i}\right)$

Net $F_{y, C}=\sum_{i=1}^{n} \mathrm{~F}_{\mathrm{C}_{\mathrm{i}}} \sin \left(\theta_{i}\right)$

Net $M_{z, C}=\sum_{i=1}^{n}-\mathrm{F}_{\mathrm{C}_{\mathrm{i}}} \cos \left(\theta_{i}\right) \cdot y_{C_{i}}+$ $F_{C_{i}} \sin \left(\theta_{i}\right) \cdot x_{C_{i}}$

Based on the above equations, all the forces and moments are summed vectorially, to get acceleration of the rotors which are integrated into the Motion module which will be discussed in Section 2.4.

Once the normal contact forces at each location are evaluated, the friction coefficient is evaluated assuming Elasto-Hydrodynamic Lubrication (EHL) at the contact points. The relation published by Hamrock [11] is used:

$\overline{\mu_{1}}=0.67 \cdot 10^{-6} \cdot\left(U^{*}\right)^{0.81} \cdot U^{0.26} \cdot(W \cdot$

$\left.G^{2}\right)^{3.32}$ when $\overline{\mu_{1}} \leq 0.8 \gamma$

$\bar{\mu}=0.8 \gamma+0.021 \cdot \tanh \left(\frac{\overline{\mu_{1}}}{\gamma}-0.8\right)$

when $\overline{\mu_{1}}>0.8 \gamma$ 


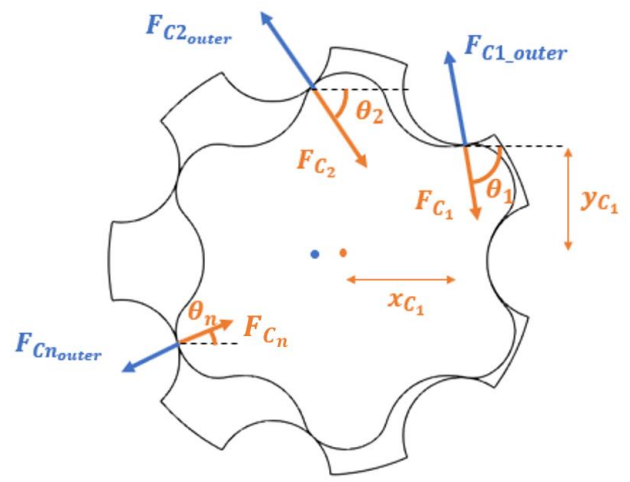

Figure 7: Illustration of forces acting on the gear

\subsection{CFD-Gap Module}

The CFD-Gap Module solves the Reynolds equation in the lubricating interfaces presented earlier (Fig. 3). The radial gap acts like a journal bearing generating resistive loads to support the outer rotor. The CFD-Gap module solves the Reynolds equation by defining a generic polar grid for the two surfaces. The general Reynolds equation where the fluid film is enclosed by a bottom surface "a" and a top surface " $b$ " is written as follows [16]:

$\frac{\partial}{\partial x}\left(\frac{\rho h^{3}}{12 \mu} \frac{\partial p}{\partial x}\right)+\frac{\partial}{\partial y}\left(\frac{\rho h^{3}}{12 \mu} \frac{\partial p}{\partial y}\right)=\frac{\partial}{\partial x}\left(\frac{\rho h\left(u_{a}+u_{b}\right)}{2}\right)+$

$\frac{\partial}{\partial y}\left(\frac{\rho h\left(v_{a}+v_{b}\right)}{2}\right)+\rho\left(w_{a}+w_{b}\right)-\rho u_{a} \frac{\partial h}{\partial x}-$

$\rho v_{a} \frac{\partial h}{\partial y}+h \frac{\partial \rho}{\partial t}$

The Reynolds equation approximates the full Navier-Stokes equations when considering thin fluid films. The boundary conditions are pressures set by each tooth space volume for the lateral gap. And for the radial gap, wall boundary conditions are applied which is proved to be a good assumption as proved by Pellegri et al. [15]. Based on these boundary conditions, the CFDGap module applies forces and moments to the gears and outputs flows into and out of the different fluid domain.

\subsection{Motion Module}

The motion module evaluates the position of the gears based on the forces and moments acting on the gears from the force module, fluid-dynamic module and the gap module using Newton's six degree of motion dynamics equations, where the forces and moments acting on the gears gives acceleration of the gear and angular acceleration of each gear with respect to its center of mass. These accelerations are integrated to get velocities and angular velocities which are further integrated to get the position of the gears for the next time step.

\subsection{Geometric Module}

The geometric module is an essential preprocessor for all the modules of the simulation tool. This module evaluates features that are necessary for evaluating fluid-dynamic properties of a Gerotor i.e. DC volume, porting areas, etc. This module is entirely based on numerical calculation performed on the CAD files (in TXT or STL format) of rotors and of the inlet and outlet ports. Therefore, the model does not require a specific analytical definition of the rotor profiles. The output of the model is given in the form of TXT files that constitute the input for the HYGESim tool. This text file contains the geometrical features, as a function of the rotation angle of the rotors. All the essential features of the geometric preprocessor module are run for the varied position of the outer gear and features such as the tooth space volumes, porting areas are output into a text file.

\subsection{Mechanical Module}

The mechanical module is also one of the preprocessor modules used for accessing contact loads on the Gerotor. Due to radial clearances present between the outer rotor and the body, the outer gear has some degree of freedom in the planar direction which needs to be accounted for.

The mechanical module takes as input the profile of the inner and outer gear of a Gerotor and based on that evaluates the contact forces. It simulates various positions of the outer gear with varying planar displacement and angle micromotion. In this simulation, there could be cases when at a certain position the outer gear profile (which is defined by points) can intersect the inner gear profile. At these locations, the module evaluates the penetration depth. Gap heights are evaluated if there aren't any intersections. This is illustrated by Fig. 8.

The contact forces are evaluated based on the research article published by Pereira C. [12], where the contact is modeled locally as an 
intersection of two cylinders and depends on the local radius of curvature and the penetration depth. The following equation is used:

$F_{C}=\frac{(a \Delta R+b) L E^{*}}{\Delta R} \delta^{n}\left[1+\frac{3\left(1-c_{r}^{2}\right)}{4} \frac{\dot{\delta}}{\dot{\delta}^{(-)}}\right]$

Where $\mathrm{a}$ and $\mathrm{b}$ are constants described in the paper [12], $c_{r}$ represents the coefficient of restitution. $\delta$ represents the depth of penetration, $\dot{\delta}$ represent the penetration velocity and $\dot{\delta}^{(-)}$is the impact velocity.

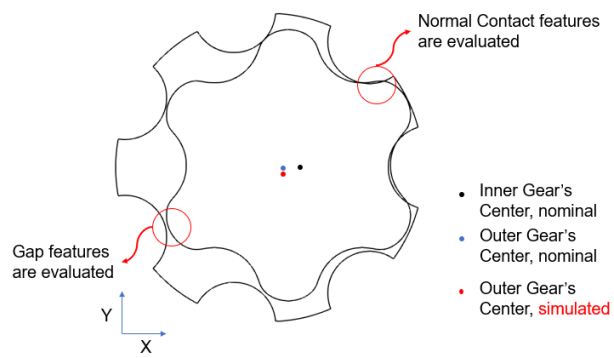

Figure 8: Gap height and Normal Contact feature evaluation

\section{EXPERIMENTAL VALIDATION}

In this section, results obtained by the proposed modelling scheme are compared with experimental measurements for the unit shown in Fig. 7. Figure 9 shows the profile of the inner and the outer gear of a $6 / 7$ tooth cycloidal gearset with suction and delivery ports shown in red and blue respectively which was used as a prototype unit for testing by Thomas Magnete $\mathrm{GmbH}$. The schematic of the experimental setup is shown in Fig. 10. An orifice connected to a line was used in the experiment as a loading element. This helped to easily reproduce pressure pulsations for validation of the model. The measurements were taken for steady-state operating conditions. A comprehensive result showing the validation of pressure as well as torque ripple is shown in the following paragraphs. The units of all the results have been normalized for confidentiality reasons.

The model is simulated with a radial clearance between outer rotor and housing, to match the clearance in the experiments which allow for micro-motions of the outer gear. Experiments were performed at various shaft speeds and pressure differentials. The case with $19.18 \%$ of the maximum tested shaft speed $\left(n_{\max }\right)$ and the maximum tested pressure drop $\left(\Delta p_{\max }\right)$ is considered for illustration purposes.

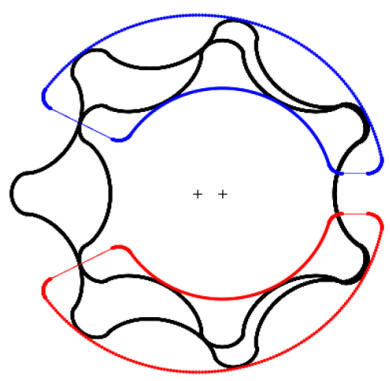

Figure 9: Cycloidal Gerotor Gear
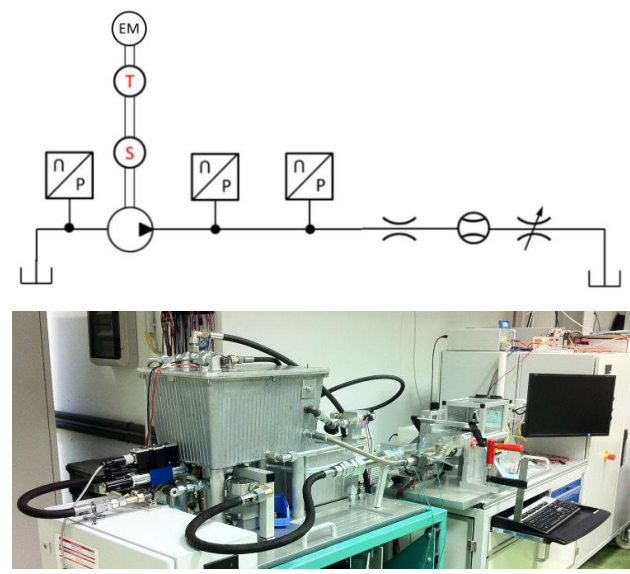

Figure 10: ISO schematic and Test Rig

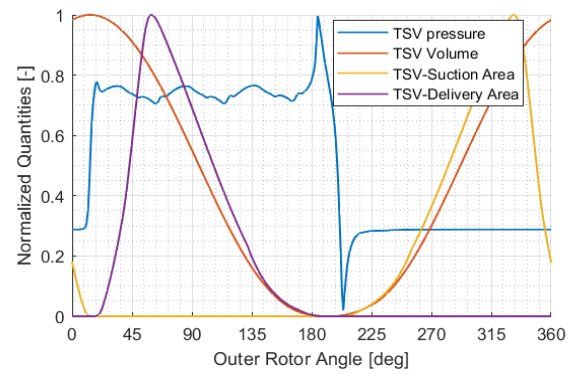

Figure 11: Normalized TSV pressure, volume and porting area with a rotational angle of the outer rotor

Figure 11 shows different normalized quantities for one of the TSV with respect to the angle of the outer rotor. The TSV expands when connected to the suction while it contracts when connected to delivery, thus displacing fluid. The figure also shows pressurization and depressurization of a TSV. 


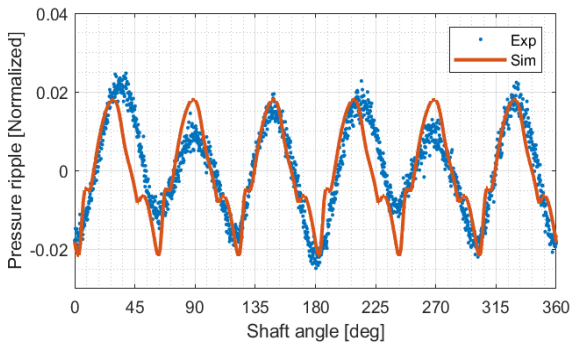

Figure 12: Comparison between experimental and simulated pressure ripple

Figure 12 shows the pressure ripple behavior of the pump at the chosen operating condition. Based on this, there is a very close match of the magnitude of the pressure ripple between the experimental results and the simulated.

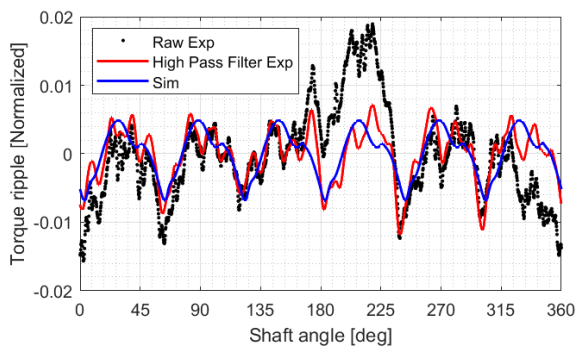

Figure 13: Comparison of torque ripple data of experiment vs simulated

Figure 13 shows a comparison of torque ripple between experimental data and simulated. The raw experimental data was filtered using a high pass filter to avoid any effects of shaft whirling or improper centering of the shaft. Once, the whirl frequency if removed, a very close match in the actual torque ripple magnitude is obtained. The source for the peak for raw experimental data in the range of $180^{\circ}$ to $225^{\circ}$ is due to the shaft misalignment.

Figure 14 shows a comparison of the torque obtained by simulation with ideal torque, and when the subsequent contribution of Gaps (radial and lateral) with contact friction following at the very end. These plots are also compared with the single contact point theory. It can be observed that in this operating condition the Gaps have a huge impact on the overall torque followed by the EHL effects at the contact location. The final torque is also compared with the single contact point assumption which ascertains that when evaluating torque ripple multiple contact points must be taken into consideration.

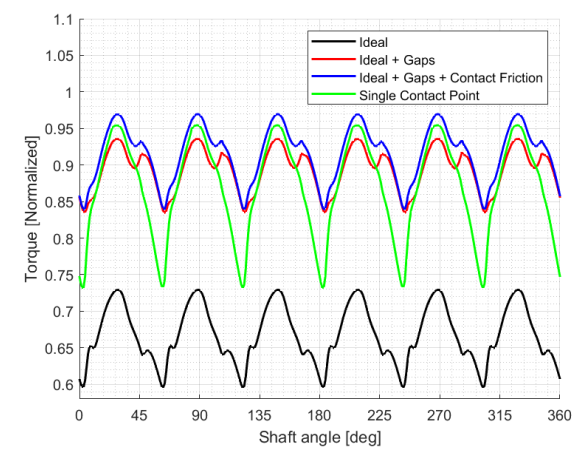

Figure 14: Distribution of torque, showing torque losses due to Gaps and Contact Friction and comparing with single contact point theory

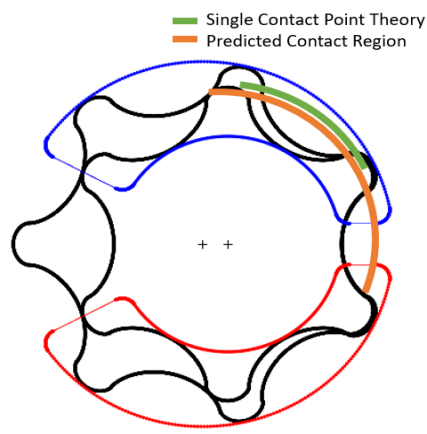

Figure 15: Angular span of predicted contact region versus single contact point theory

Figure 15 shows a comparison between the predicted angular region of contact by the current model versus single contact point theory. It can be observed that the predicted angular region of contact is nearly twice as that of the single contact point theory.

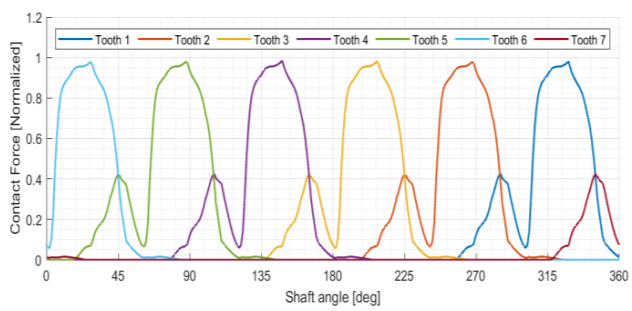

Figure 16: Contact forces by each tooth with respect to shaft angle 
Figure 16 shows the variation of the contact force by each tooth. It is observed that at any shaft angle the contact force is applied by at least two different teeth and sometimes three. Therefore, this gearset has two contact points for the operating condition.

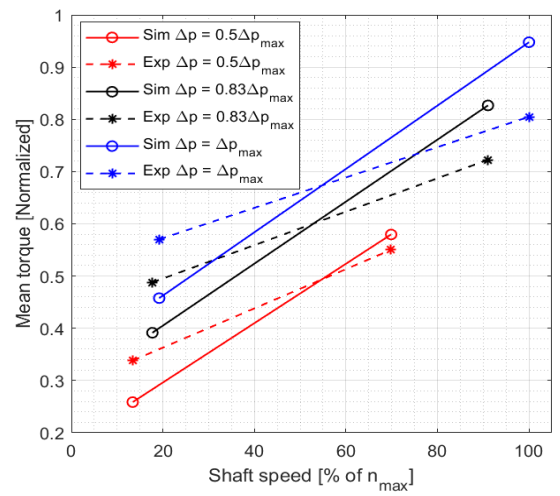

Figure 17: Comparison of mean torque with different operating conditions

Figure 17 compares the mean torque of experimental setup and simulated at different operating conditions. It can be observed that with an increase in rotational speed keeping the same pressure drop increases the mean torque. This general trend is matched by the simulation. But there are discrepancies which could have been caused by the asymmetric behavior of the lateral gaps which are on either side of the gears which is not accounted for in this study.

\section{CONCLUSION}

This paper presented a novel formulation of a Gerotor modeling tool. The Gerotor model is based on a fast lumped-parameter based approach which discretizes the Gerotor into a set of tooth space volumes that have the same properties inside a lumped volume. The model also considers the possibility of multiple contact points that drive the outer rotor with the presence of the radial gap that supports the outer rotor. The model solves for the Reynolds equation for the lubricating interfaces and Elasto-Hydrodynamic Lubrication (EHL) at the contact locations. It also solves for instantaneous micro-motion of the gears inside the body.

The results of the simulation are compared against that of the experiments. Within the research, experiments were performed for a $6 / 7$ tooth Cycloidal prototype Gerotor unit. Steadystate tests performed with the experiments allowed a comparison of simulation and experiment.

The results show a good correlation between the simulations and experiments outlet pressure pulsations. With the inclusion of multiple contact points, friction at the contact points and radial gap for the outer rotor, a good correlation is obtained for the shaft torque ripple.

\section{NOMENCLATURE}

$\begin{array}{cl}A & \text { Area, } m^{2} \\ E & \text { Modulus of Elasticity, } N / m^{2} \\ \text { EHL } & \text { Elasto-Hydrodynamic Lubrication } \\ E^{\prime} & \text { Effective elastic modulus, } \frac{2}{\left[\frac{1-v_{a}^{2}}{E_{a}}+\frac{1-v_{b}^{2}}{E_{b}}\right]}, N / \mathrm{m}^{2} \\ & \\ F & \text { Force, } \mathrm{N} \\ F_{C} & \text { Contact Force, } \mathrm{N} \\ G & \text { Dimensionless Material Parameter, } \alpha E^{\prime} \\ R & \text { The radius of Curvature, } \mathrm{m} \\ R_{e q} & \text { Equivalent Radius of Curvature, } \frac{1}{\frac{1}{R_{a}}+\frac{1}{R_{b}}}, \mathrm{~m} \\ T S V & \text { Tooth Space Volume } \\ U^{*} & \text { Dimensionless sliding velocity, } u_{d} / u_{s} \\ U & \text { Dimensionless speed parameter, } \mu_{0} u_{s} / E^{\prime} R \\ W & \text { Dimensionless load parameter, } F_{s} / b E^{\prime} R \\ & \\ b & \text { The face width of the Gears, } \mathrm{m} \\ h & \text { Height, m } \\ h_{t} & \text { Height of the tooth tip gap, } \mathrm{m} \\ p & \text { Pressure, Pa } \\ u & \text { Velocity in x-direction, } \mathrm{m} / \mathrm{s} \\ u_{d} & \text { Velocity difference, } u_{a}-u_{b}, \mathrm{~m} / \mathrm{s} \\ u_{s} & \text { Velocity sum, } u_{a}+u_{b}, \mathrm{~m} / \mathrm{s} \\ v & \text { Velocity in y-direction, } \mathrm{m} / \mathrm{s} \\ w & \text { Velocity in z-direction, } \mathrm{m} / \mathrm{s} \\ \mu & \text { Friction Coefficient } \\ \mu_{0} & \text { Absolute Viscosity } \\ \gamma & \text { Limiting-shear-strength proportionality constant } \\ v & \text { Poisson's ratio } \\ \rho & \text { Density, } k g / m^{3} \\ & \text { Rotational Speed, rad/sec } \\ & \\ & \end{array}$

\section{REFERENCES}

[1] Bonandrini G, Mimmi G, Rottenbacher C (2009) Theoretical analysis of internal epitrochoidal and hypotrochoidal machines. Proc Inst Mech Eng Part C J Mech Eng Sci 223(6):1469-1480 
[2] Mimmi G, Bonandrini G, Rottenbacher C (2007) Theoretical analysis of internal lobe pumps. IFToMM World Congress

[3] Manco S, Nervegna N, Rundo M (2002) A contribution to the design of hydraulic lube pumps. Int J Fluid Power 3(1):21-32

[4] Manco S, Nervegna N, Rundo M, Torino P, Armenio G, Pachetti C, Trichilo R Gerotor lubricating oil pump for ic engines (1998) SAE Paper 982689, International fall fuels and lubricants meeting and exposition, San Francisco, USA and SAE Transactions

[5] Schweiger W, Schoefmann W, Vacca A (2011) Gerotor pumps for automotive drivetrain applications: a multi-domain simulation approach GRPs in AWD-powertrain systems. SAE Int J Passeng Cars Mech Syst 4(3):13581376. DOI:10.4271/ 2011-01-2272

[6] Gamez-Montero P-J, Castilla R, del Campo D, Erturk N, Raush G, Codina E (2012) Influence of the inner teeth clearances on the flow ripple in a gerotor pump for engine lubrication. Proc Inst Mech Eng Part D J Automob Eng 226(7):930942

[7] Altare G, Rundo M (2015) CFD analysis of gerotor lubricating pumps at high speed: geometric features influencing the filling capability. ASME/BATH Conf 2015:1-10

[8] Pellegri M, Vacca A, Frosina E, Buono D, Senatore A (2017) Numerical analysis and experimental validation of Gerotor pumps: A comparison between a lumped parameter and a computational fluid dynamics-based approach. Proc IMechE Part C Vol. 231(23) 4413-4430

[9] Pelligri M, Vacca A (2017) Numerical simulation of Gerotor pumps considering rotor micro-motions. Meccanica (2017) 52:18511870

[10] Gamez-Montero PJ, Castilla R, Khamashta M, Codina E (2006) Contact problems of a trochoidal-gear pump. Int J Mech Sci 48:14711480

[11] Jacobson B O, Hamrock B J (1984) NonNewtonian Fluid Model Incorporated Into Elastohydrodynamic Lubrication of Rectangular Contacts. J. Tribol. Apr 1984, 106(2): 275-282

[12] Pereira C., Ramalho A., Ambrosio J. (2015) An Enhanced Cylindrical Contact Force Model, J. Multibody Syst Dyn, 35: 277

[13] LMS Imagine SA (2015) Hydraulic library, User Manual, Rev 14

[14] Rituraj F, Vacca A. (2019) Modelling and Validation of Tooth Tip Leakages in Gerotor
Pumps. Proceedings of the ASME-JSME-KSME 2019 Joint Fluids Engineering Conference AJKFLUIDS2019

[15] Pellegri M, Vacca A (2017) A Simulation Approach for the Evaluation of Power Losses in the Axial Gap of Gerotor Units. JFPS International Journal of Fluid Power System 11$3,55 / 62,2018$

[16] Reynolds O (1886) On the Theory of Lubrication and Its Application to $\mathrm{Mr}$. Beauchamp Tower's Experiments, Including an Experimental Determination of the Viscosity of Olive Oil. Philosophical Transactions of the Royal Society of London. Royal Society. 177: 157-234. doi:10.1098/rstl.1886.0005. JSTOR 109480 